\title{
Analysis of the Voltage Losses in CZTSSe Solar Cells of Varying Sn Content
}

\author{
Mohammed Azzouzi, ${ }^{\dagger, \# \oplus ~ A n t o n i o ~ C a b a s-V i d a n i, ~}{ }^{\ddagger}, \#$ Stefan G. Haass, ${ }^{\ddagger}$ Jason A. Röhr, ${ }^{\dagger}$ \\ Yaroslav E. Romanyuk, Ayodhya N. Tiwari, and Jenny Nelson*, ${ }^{\ddagger}$ \\ ${ }^{\dagger}$ Department of Physics and Centre for Plastic Electronics, Imperial College London, London SW7 2AZ, United Kingdom \\ ${ }^{\ddagger}$ Laboratory for Thin Films and Photovoltaics, Empa-Swiss Federal Laboratories for Materials Science and Technology, \\ Ueberlandstrasse 129, 8600 Duebendorf, Switzerland
}

\section{Supporting Information}

ABSTRACT: The performance of kesterite $\left(\mathrm{Cu}_{2} \mathrm{ZnSn}\right.$ $\left.(\mathrm{S}, \mathrm{Se})_{4}, \mathrm{CZTSSe}\right)$ solar cells is hindered by low open circuit voltage $\left(V_{\text {oc }}\right)$. The commonly used metric for $V_{\text {oc }}$-deficit, namely, the difference between the absorber band gap and $q V_{\text {oc }}$, is not well-defined for compositionally complex absorbers like kesterite where the bandgap is hard to determine. Here, nonradiative voltage losses are analyzed by measuring the radiative limit of $V_{\text {oc }}$ using external quantum efficiency (EQE) and electroluminescence (EL) spectra, without relying on precise knowledge of the bandgap. The method is applied to a series of $\mathrm{Cu}_{2} \mathrm{ZnSn}(\mathrm{S}, \mathrm{Se})_{4}$ devices with Sn content variation from 27.6 to 32.9 at. \% and a corresponding $V_{\text {oc }}$ range from 423 to $465 \mathrm{mV}$. Surprisingly,

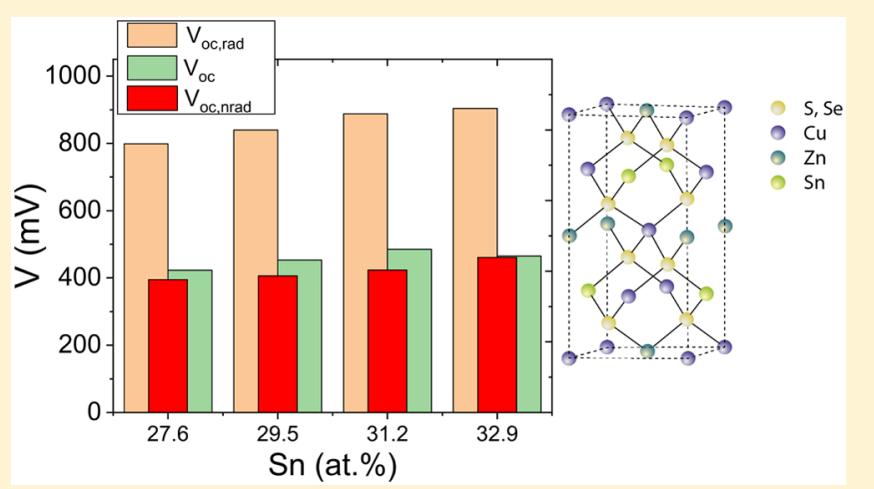
the lowest nonradiative loss, and hence the highest external luminescence efficiency $\left(\mathrm{QE}_{\mathrm{LED}}\right)$, were obtained for the device with the lowest $V_{\text {oc }}$. The trend is assigned to better interface quality between absorber and CdS buffer layer at lower Sn content.

K esterite solar cells are a promising earth-abundant alternative to existing thin film photovoltaic technologies. ${ }^{1}$ Even though their power conversion efficiencies (PCE) have increased significantly during the past decade from $4 \%$ in 2004 to $12.6 \%$ in $2014,{ }^{2}$ they still lie far below those of competing technologies like $\mathrm{Cu}(\mathrm{In}, \mathrm{Ga}) \mathrm{Se}_{2}$ and $\mathrm{CdTe}$ that have surpassed $22 \%{ }^{3}$ One of the major issues is the significant $V_{\text {oc }}$ deficit in these devices defined relative to the bandgap $E_{\mathrm{g}}$ of the absorber or relative to the $V_{\text {oc }}$ in the Shockley-Queisser limit for the same band gap $\left(V_{\mathrm{oc}, \mathrm{SQ}}\left(E_{\mathrm{g}}\right)\right){ }^{4}$ Kesterite devices show a deficit $\left(E_{\mathrm{g}} / q-V_{\text {oc }}\right)$ larger than $0.55 \mathrm{~V}$ whereas competing technologies have reduced these losses to less than $0.4 \mathrm{~V}^{5}$ In the literature, the lowest values of $V_{\text {oc }}$ deficit in devices have been obtained by means of interfacial and compositional optimization $^{6,7}$ with the highest achieved $V_{\text {oc }}$ relative to the band gap of a kesterite solar cell being still $0.4 \mathrm{~V}$ lower than $V_{\text {oc,SQ. }}{ }^{8}$

Several mechanisms have been investigated as the origin for the large voltage losses in CZTSSe solar cells. ${ }^{9-11}$ A primary mechanism is thought to be related to the high defect density and associated band tailing of CZTSSe materials, ${ }^{12,13}$ which are attributed to the multielement composition nature of the quaternary CZTSSe phase. The similar ionic radii, and comparable valences of elements like copper and zinc, lead to a narrow chemical stability region and multiple defects with low activation energies. ${ }^{14}$ The best performing devices have an off-stoichiometric absorber composition, more specifically $\mathrm{Cu}$ - poor and $\mathrm{Zn}$-rich. This reduces the formation of detrimental defects like interstitials $\left(\mathrm{Cu}_{\mathrm{i}}\right.$ and $\left.\mathrm{Zn}_{\mathrm{i}}\right)$ and $\left[2 \mathrm{Cu}_{\mathrm{Zn}}+\mathrm{Sn}_{\mathrm{Zn}}\right]$ antisite clusters that adversely affect the solar cell performance, while increasing the concentration of copper vacancies $\left(\mathrm{V}_{\mathrm{Cu}}\right)$, which is a beneficial shallow acceptor. ${ }^{15,}$

It has been shown that CZTSSe absorbers and related sulfur-selenium alloys crystallize in the kesterite-type structure where the band gap varies from $1.0 \mathrm{eV}$ for pure selenide (CZTSe) to $1.5 \mathrm{eV}$ for pure sulfurized (CZTS), opening the possibility to tailor the band gap of kesterite through the S-Se content. Additionally, the structural instability due to the low enthalpy cost of swapping $\mathrm{Cu}$ and $\mathrm{Zn}$ atoms in kesterites has also been shown to affect the band gap. ${ }^{17}$ In fact, the ordered CZTSe $\left(\mathrm{Cu}_{2} \mathrm{ZnSnSe}_{4}\right)$ has a band gap $100 \mathrm{meV}$ higher than the disordered one. ${ }^{18}$ Márquez et al. observed that $\mathrm{Cu}$-poor CZTSe had a higher band gap and $V_{\text {oo }}$ linked to an increased ordering of the $\mathrm{Cu} / \mathrm{Zn}$ sublattice, while the $V_{\mathrm{oc} \text {,def }}$ remained constant. $^{19}$ Similarly, through annealing procedures with increasing temperatures and subsequent rapid cool-down, a reversible order-disorder transition was shown to occur at a critical temperature of about $200{ }^{\circ} \mathrm{C}$, leading to a band gap shift equivalent to the one observed with the change in $\mathrm{Cu}$ content. $^{20}$

Received: February 21, 2019

Accepted: May 9, 2019

Published: May 9, 2019 
The strong dependence of the band gap of kesterite absorbers on their composition and processing condition, coupled with the complexity of quantifying the band gap energy of a disordered system such as CZTSSe, ${ }^{21}$ raises the question of whether a $V_{\text {oc }}$ loss analysis solely related to the band gap energy gives relevant information concerning the origin of the losses and their dependence on material properties. From the reciprocity principle and the measured external quantum efficiency, Rau et al. ${ }^{22}$ introduced a radiative limit for the open circuit voltage that incorporates the actual absorption tail of the materials, as expressed via the external quantum efficiency (EQE) spectrum. On the basis of this work, Yao et al. introduced another way to quantify the losses in solar cells, ${ }^{23}$ where they differentiated the losses due to an extended absorption onset $\left(\Delta V_{\text {oc,abs }}\right)$ from the losses due to nonradiative recombination $\left(\Delta V_{\mathrm{oc}, \mathrm{nrad}}\right)$, that are dependent on the ratio of radiative to nonradiative recombination rates. This approach was used on organic solar cells to distinguish nonradiative losses from the total losses to indicate how changes in material or processing could bring $V_{\text {oc }}$ closer to the radiative limit. ${ }^{24,25}$

In this work we focus on a series of CZTSSe solar cells where compositional variation, namely, the Sn content, was shown to affect $V_{\text {oc }}$ as well as the band gap energy of the absorber in a similar manner to the ordering effect of the $\mathrm{Cu}$ / Zn sublattice. We first show how a method relying solely on $E_{\mathrm{g}}$ to quantify the $V_{\mathrm{oc}}$ deficit depends strongly on the way $E_{\mathrm{g}}$ is calculated. We then use the method described above developed by Yao et al. ${ }^{23}$ to calculate the radiative limit of the voltage $\left(V_{\text {oc,rad }}\right)$. This approach has never been applied to CZTSSe absorbers, but it proves to be very relevant, since the bandgap cannot be reliably determined by traditional methods, due to material complexity. ${ }^{21}$ Surprisingly, we find that this $V_{\text {oc }}$ increase with $\mathrm{Sn}$ content was accompanied by an increase rather than a decrease of $\Delta V_{\text {oc,nrad }}$ indicating a negative effect of the $\mathrm{Sn}$ content on the nonradiative relative to radiative recombination rate. By measuring both temperature dependent current voltage characteristic (JV-T) and temperature dependent capacitance-frequency $(\mathrm{Cf}-\mathrm{T})$, we discard the hypothesis that this improvement is due to a change in the defect distribution in the absorber structure and show that it can be related to the interfacial quality between the absorber and the buffer layers. The type of analysis presented here is applied to further characterize the different recombination mechanisms that limit the $V_{\text {oc }}$ of CZTSSe and other devices and identify optimization routes to increase $V_{\text {oc }}$.

Voltage Loss Calculation Method. For solar cells, $V_{\mathrm{oc}}$ is defined by the balance between the absorbed photon flux and the recombination flux. In other words, the recombination current density $J_{\text {rec }}$ and the photocurrent density $J_{\mathrm{ph}}$ are equal at open circuit, $J_{\mathrm{rec}}\left(V_{\mathrm{oc}}\right)=J_{\mathrm{ph}}\left(V_{\mathrm{oc}}\right)$. Using the nonideal diode equation we can typically express $V_{\text {oc }}$ as

$$
V_{\mathrm{oc}}=\frac{n_{\mathrm{id}} k_{\mathrm{B}} T}{q} \ln \left(\frac{J_{\mathrm{ph}}\left(V_{\mathrm{oc}}\right)}{J_{0}}+1\right)
$$

where $n_{\mathrm{id}}$ is the ideality factor, $k_{\mathrm{B}}$ is the Boltzmann constant, $q$ is the elementary charge, and $J_{0}$ is the dark saturation current density (neglecting any effect of shunt resistance at open circuit). Both $J_{0}$ and $n_{\mathrm{id}}$ depend on the dominant recombination process. The Shockley-Queisser (SQ) limit for the open-circuit voltage is similarly given by

$$
V_{\mathrm{oc}, \mathrm{SQ}}=\frac{k_{\mathrm{B}} T}{q} \ln \left(\frac{J_{\mathrm{ph}, \mathrm{SQ}}\left(V_{\mathrm{oc}}\right)}{J_{0, \mathrm{SQ}}}+1\right)
$$

where $J_{\mathrm{ph}, \mathrm{SQ}}$ is the photocurrent density in the radiative limit and $J_{0, S Q}$ is the SQ dark saturation current density. In practice, $J_{\mathrm{ph}, \mathrm{SQ}}\left(V_{\mathrm{oc}}\right)$ can be replaced by the measured short-circuit current density $\left(J_{\text {sc }}\right)$ at the corresponding light intensity. As discussed in ref 23, this assumption introduces a small error in the calculation of the open circuit voltages of some millivolts that can be neglected. The $J_{0, S Q}$ is obtained directly from the band gap $E_{\mathrm{g}}$ following the detailed balance of absorption and emission in solar cell as expressed by Shockley and Queisser: ${ }^{4}$

$$
J_{0, \mathrm{SQ}}=q \int_{E_{\mathrm{g}}}^{\infty} \phi_{\mathrm{BB}}(E) \mathrm{d} E
$$

where a step function for the external quantum efficiency at the band gap is assumed. $\phi_{\mathrm{BB}}$ is the spectral blackbody emission flux density in the forward direction integrated from the surface of the blackbody at the cell temperature $T_{\mathrm{c}}$

$$
\phi_{\mathrm{BB}}(E, T)=2 \pi \times \frac{E^{2}}{h^{3} c^{2}} \frac{1}{\left(\exp \left(\frac{E}{k_{\mathrm{B}} T_{\mathrm{c}}}\right)-1\right)}
$$

which is expressed in units of $\mathrm{cm}^{-2} \mathrm{~s}^{-1} \mathrm{eV}^{-1}$. Here $h$ is Planck's constant and $c$ is the speed of light in vacuum (we consider the refractive index of the external medium surrounding the cell to be $\left.n_{\mathrm{s}}=1\right) .{ }^{26}$ Using a similar approach, but considering the actual quantum efficiency of the cell $(E Q E(E))$, we can express the radiative dark saturation current $J_{0, \text { rad }}$ as the integral of the EL emission from the cell

$$
J_{0, \mathrm{rad}}=q \int \phi_{\mathrm{EL}}(E) \mathrm{d} E=q \int \operatorname{EQE}(E) \phi_{\mathrm{BB}}(E) \mathrm{d} E
$$

where $\phi_{\mathrm{EL}}$ is the spectral photon flux density emitted by the cell. We used Rau's reciprocity principle to write $\phi_{\mathrm{EL}}$ as $\mathrm{EQE} \phi_{\mathrm{BB}}$ for the cell at equilibrium. ${ }^{22}$ Away from equilibrium, the excess electroluminescence emission $\left(\delta \phi_{\mathrm{em}}(E)\right)$ is related to the internal voltage $V_{\text {int }}$ experienced by the cell

$$
\delta \phi_{\mathrm{em}}(E)=\operatorname{EQE}(E) \phi_{\mathrm{BB}}(E, T)\left[\exp \left(\frac{q V_{\text {int }}}{k_{\mathrm{B}} T}\right)-1\right]
$$

Thus, we can calculate $\operatorname{EQE}(E)$ by measuring $\delta \phi_{\mathrm{em}}(E)$ at different injection currents and by using the known form of $\phi_{\mathrm{BB}}(E, T)$. Since electroluminescence can be measured at deeper photon energies in the tail of absorption than photocurrent, we can use the relation between $\delta \phi_{\mathrm{em}}(E)$ and $\mathrm{EQE}(E)$ to extend the quantum efficiency spectrum to lower energy by several tenths of an electronvolt beyond the range that is accessible through direct electrical measurement. In practice, it is difficult to quantify the internal voltage of the cell at fixed injection current. Therefore, the $\operatorname{EQE}(E)$ spectrum extracted from EL at any applied bias is scaled by a constant factor in order to coincide with edge of the measured quantum efficiency spectrum using a calibrated electrical setup. Using the extended quantum efficiency, measured in this way, we can calculate the achievable voltage if only radiative recombination occurs,

$$
V_{\mathrm{oc}, \mathrm{rad}}=\frac{k_{\mathrm{B}} T}{q} \ln \left(\frac{J_{\mathrm{sc}}}{J_{0, \mathrm{rad}}}+1\right)
$$



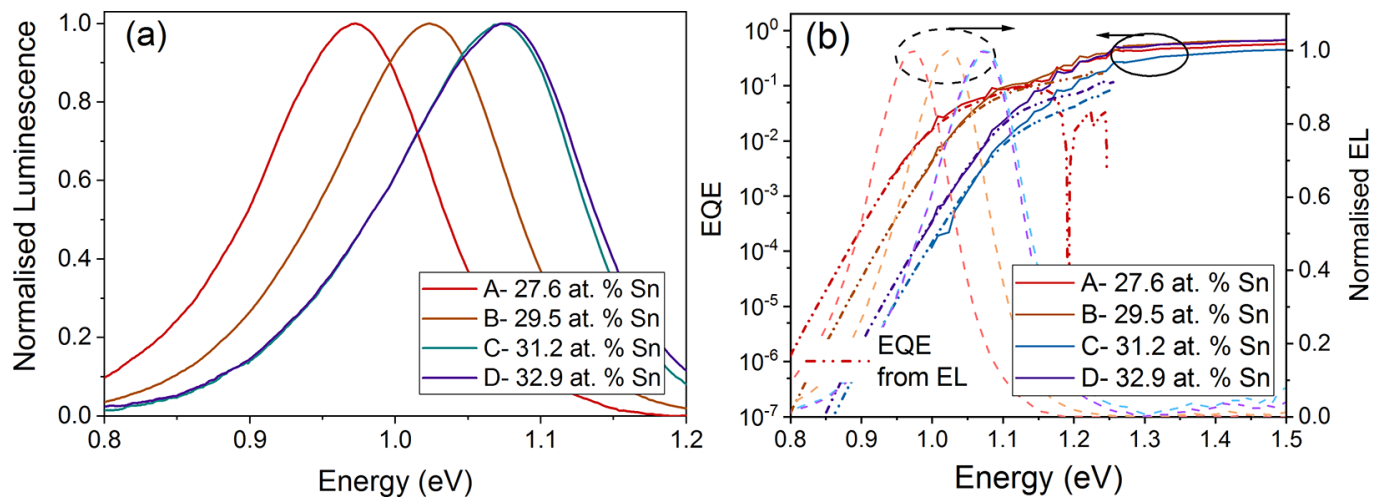

Figure 1. (a) Normalized EL spectra for the four different cells. All EL spectra were acquired using an injection current density of $40 \mathrm{~mA} \mathrm{~cm}{ }^{-2}$. (b) Measured EQE for the four cells (bold lines) and the extended EQE reconstructed one from their EL spectra (short dashed lines). The full PL spectra are presented in the Supporting Information, Figure S10.

where we use the fact that $n_{\mathrm{id}}=1$ for radiative recombination and let $J_{0}=J_{0, \text { rad }}$ in the radiative limit. We can now express the excess voltage losses due to the dominating nonradiative losses $\operatorname{as}^{22,23}$

$$
\begin{aligned}
\Delta V_{\mathrm{oc}, \mathrm{nrad}} & =V_{\mathrm{oc}, \mathrm{rad}}-V_{\mathrm{oc}}=-\frac{k_{\mathrm{B}} T}{q} \ln \left(\frac{J_{0, \mathrm{rad}}}{J_{0}}\right) \\
& =-\frac{k_{\mathrm{B}} T}{q} \ln \left(\mathrm{QE}_{\mathrm{LED}}\left(V_{\mathrm{oc}}\right)\right)
\end{aligned}
$$

where $\mathrm{QE}_{\mathrm{LED}}\left(V_{\mathrm{oc}}\right)$ is the electroluminescence quantum efficiency of the solar cell at an applied bias of $V_{\mathrm{oc}}$. The latter expression emphasizes the link between the efficiency of a solar cell and that of a light emitting diode. Excess nonradiative voltage losses are detrimental for the efficiency of solar cells.

In the case where one can accurately measure the band gap of the absorber, we can additionally quantify the excess losses due to a nonsharp absorption onset by calculating the SQ limit for the $V_{\mathrm{oc}}\left(V_{\mathrm{oc}, \mathrm{SQ}}\right) .{ }^{4}$ The abrupt absorption edge considered in the SQ theory does not apply in the presence of sub band gap absorption that is clearly observed in CZTSSe devices. This difference allows the excess absorption open circuit voltage loss to be defined as

$$
\Delta V_{\mathrm{oc}, \mathrm{abs}}=V_{\mathrm{oc}, \mathrm{SQ}}-V_{\mathrm{oc}, \mathrm{rad}}
$$

However, for CZTSSe devices where strong band tailing complicates the calculation of the band gap, this equation of $\Delta V_{\text {oc,abs }}$ will be subject to uncertainty. Nonetheless, the use of $V_{\text {oc,rad }}$ to quantify the excess nonradiative losses gives reliable information on the nonradiative voltage losses even when $E_{\mathrm{g}}$ is uncertain.

Synthesis of Absorbers and Device Performance. Photovoltaic devices of structure glass/Mo/CZTSSe/CdS/i-ZnO/Al:ZnO/ $\mathrm{MgF}_{2}$ are prepared using CZTSSe absorbers in which the $\mathrm{Sn}$ content, measured as nominal atomic percentage (at. \%), is controlled by varying the $\mathrm{SnCl}_{2}$ concentration in the precursor solution as presented elsewhere. ${ }^{27}$ Devices A, B, C, and D correspond to CZTSSe absorbers with nominal Sn concentrations of 27.6, 29.5, 31.2, and 32.9 at. \%, respectively. For lower concentrations the formation of the $\mathrm{Cu}_{x}(\mathrm{~S}, \mathrm{Se})$ phase is observed, while the highest $\mathrm{Sn}$ content is the limit at which $\mathrm{Sn}(\mathrm{S}, \mathrm{Se})_{x}$ phases started to form. These secondary phases cause performance degradation and shunting of the solar cells. The chemical compositions of the absorber layers were determined by X-ray fluorescence (XRF) measurements
(Table $\mathrm{S} 1$ ). The $\mathrm{Cu} / \mathrm{Sn}$ ratio decreased with increasing $\mathrm{Sn}$ content as expected, while the ratios $\mathrm{Cu} /(\mathrm{Zn}+\mathrm{Sn})$ and $\mathrm{Zn} / \mathrm{Sn}$ are in the $\mathrm{Cu}$-poor and $\mathrm{Zn}$-rich regions compared to stoichiometric $\mathrm{Cu}_{2} \mathrm{ZnSn}(\mathrm{S}, \mathrm{Se})_{4} \cdot{ }^{15,16} \mathrm{X}$-ray diffraction measurements (Figure $\mathrm{S} 1 \mathrm{~b}$ ) confirm decreasing disorder of the $\mathrm{Cu} / \mathrm{Zn}$ sublattice with $\mathrm{Sn}$ increasing content, through the shift of the 008 peak toward higher $2 \theta$ values (further details are presented in the Supporting Information). However, such a shift could also be caused by an overlap of different kinetics due to change in composition. Therefore, additional experiments would be required to verify this interpretation.

Current density-voltage (JV) characteristics under AM1.5G simulated solar irradiation show an increase in $V_{\text {oc }}$ with $\mathrm{Sn}$ content until 31.2 at. \% (device C) and a subsequent drop for device D (Table 2 and Figure S2). The poor performance of device $\mathrm{D}$ appears to be caused by the formation of a $\mathrm{Sn}(\mathrm{S}, \mathrm{Se})_{x}$ secondary phase, as shown with XRD measurements (Figure S1a), whereas this phase is not detected for the rest of the series. Overall, the PCEs of these devices follow a trend comparable to that of $V_{\text {oc }}$ (Figure S2) with the highest efficiency achieved for device $C$ with a PCE of $8.3 \%$. Larramona et al. ${ }^{28}$ also observed the existence of an optimal $\mathrm{Sn}$ concentration for device performance, above which increasing Sn content causes a reduction in efficiency due to the formation of secondary phases.

Emission and Absorption Properties. In order to understand the origin of the higher $V_{\mathrm{oc}}$ in device $\mathrm{C}$ compared to the other devices, we proceeded to measure EL, photoluminescence (PL), and sub-bandgap EQE spectra for all devices. Figure 1a presents their EL spectra, which originate from photons emitted during recombination of the injected or photoexcited charge carriers, respectively. We note that the slight red-shifted emission of the EL emission compared to the PL emission can be attributed to the Moss-Burstein effect ${ }^{29}$ (Figure S10 for the $\mathrm{PL}$ ). Significantly, when increasing the Sn content from 27.6 to 32.9 at. \% a blue shift in both the EL and PL peaks is observed. The strong blue shift in the luminescence upon increasing $\mathrm{Sn}$ content suggests either an increase in the band gap of the material, probably related to a reduction in disorder of the kesterite crystal, ${ }^{18}$ or a change in properties of emissive defects close to the band edges. For example, a blue shift in luminescence could, in principle, result from an increase in the density of shallow defects near the band edge, such as the $\mathrm{V}_{\mathrm{Cu}}+\mathrm{Zn}_{\mathrm{Cu}}$ defects, relative to the density of deep defects, or a decrease in the density of the $\mathrm{Zn}_{\mathrm{Cu}}+\mathrm{Cu}_{\mathrm{Zn}}$ defect. ${ }^{30,31} \mathrm{~A}$ similar 
blue shift of the band gap upon changing Sn content has been observed in such cells when decreasing the $\mathrm{Cu}$ content. ${ }^{32}$

Figure $1 \mathrm{~b}$ shows the measured EQE as well as the subbandgap EQE spectra reconstructed from the EL measurements using eq 6 . The excellent overlap of the measured EQE curve and the reconstructed EQE curve justifies the use of the method to extend the measured EQE using the luminescence measurement. ${ }^{33}$ Importantly, the same blue shift that we observed in the EL and PL with Sn content is observed in the sub-band gap EQE. Notably, the slope of the tail of the reconstructed EQE below the EL peak does not change significantly with increasing $\mathrm{Sn}$ content. If we consider a model of tail states below those band gap energies, ${ }^{34}$ this suggests that the distribution of these tail states does not change with $\mathrm{Sn}$ content.

Comparison of Voltage Losses Analysis. First, we estimate the voltage deficit in these cells by comparing the bandgap with the measured open circuit voltage, as is common in the literature. ${ }^{32}$ We estimate the band gap of the cells using two different methods, first from the inflection point of the EQE curve when plotted on a linear axes (Figure $S 4)^{13}\left(E_{\mathrm{g}, \mathrm{EQE}}\right)$, and second by fitting the EL spectrum (Figure S5,Table S2) using the method developed by Katahara et al. ${ }^{35}\left(E_{\mathrm{g}, \mathrm{EL}}\right)$. The band gaps extracted using these methods differ significantly, as shown in Table 1 ,

Table 1. Summary of the Band Gap Loss Analysis for the Four Different Devices

\begin{tabular}{ccccccc} 
label & $\begin{array}{c}\text { Sn content } \\
(\text { at. \% })\end{array}$ & $\begin{array}{c}E_{\mathrm{ggOE}} \\
(\mathrm{eV})\end{array}$ & $\begin{array}{c}E_{\mathrm{ggEL}} \\
(\mathrm{eV})\end{array}$ & $\begin{array}{c}V_{\text {oc }} \\
(\mathrm{mV})\end{array}$ & $\begin{array}{c}V_{\text {oc,def,EQE }} \\
(\mathrm{mV})\end{array}$ & $\begin{array}{c}V_{\text {oc,def,EL }} \\
(\mathrm{mV})\end{array}$ \\
$\mathrm{A}$ & 27.6 & 1.13 & 1.04 & 423 & 707 & 617 \\
$\mathrm{~B}$ & 29.5 & 1.14 & 1.07 & 453 & 687 & 617 \\
$\mathrm{C}$ & 31.2 & 1.14 & 1.11 & 485 & 655 & 625 \\
$\mathrm{D}$ & 32.9 & 1.15 & 1.12 & 465 & 685 & 655 \\
\hline
\end{tabular}

and result in very different trends in the voltage losses; if we rely on $E_{\mathrm{g}}$ from the $\mathrm{EQE}$ spectrum, the $V_{\text {oc }}$ deficit reaches a minimum for device $C$, whereas for $E_{\mathrm{g}}$ values derived from $\mathrm{EL}$ the losses are similar for the first three devices and increase only for device D (Table 1). This emphasizes the difficulty of relying on the band gap to understand the trends in voltage losses with material composition in a disordered material such as CZTSSe.

In order to obtain more reliable values for the voltage losses, we calculate $V_{\text {oc,rad }}$ of the different cells from the extended EQE spectrum. Table 2 shows the calculated values, showing that

Table 2. Summary of the Voltage Loss Analysis for the Four Cells with Different Sn Content

\begin{tabular}{cccccc} 
label & $\begin{array}{c}\text { Sn content }(\text { at. } \\
\%)\end{array}$ & $\begin{array}{c}V_{\text {oc,rad }} \\
(\mathrm{mV})\end{array}$ & $\begin{array}{c}V_{\text {oc }} \\
(\mathrm{mV})\end{array}$ & $\begin{array}{c}\Delta V_{\text {oc,nrad }} \\
(\mathrm{mV})\end{array}$ & $\mathrm{QE}_{\mathrm{LED}}(\%)$ \\
$\mathrm{A}$ & 27.6 & 799 & 423 & 376 & $5.2 \times 10^{-5}$ \\
B & 29.5 & 840 & 453 & 387 & $3.3 \times 10^{-5}$ \\
$\mathrm{C}$ & 31.2 & 888 & 485 & 403 & $1.8 \times 10^{-5}$ \\
D & 32.9 & 904 & 465 & 439 & $4.6 \times 10^{-6}$ \\
\hline
\end{tabular}

$V_{\text {oc,rad }}$ increases with increasing Sn content, where the device with the highest $S n$ content (device D) has a $V_{\text {oc,rad }}$ that is 100 meV higher than the device with the lowest Sn content (device A). The trend in $V_{\text {oc,rad }}$ is a quantitative reproduction of the observed (qualitative) blue shift in the EQE onset as well as the EL and PL peak seen in Figure 1 and can be understood as an increase in the maximum achievable $V_{\text {oc }}$ in the radiative limit of these devices with increasing Sn content. Using the two bandgap extracted previously (Table 1 ), we hence calculated $V_{\mathrm{oc}, \mathrm{SQ}}$ for the four devices and compared it to the calculated $V_{\text {oc,rad }}$ values (Table 2). From Table S3, it is clear that neither method of calculating the band gap can accurately replicate the trend in $V_{\text {oc,rad }}$.

We proceed to calculate the contribution of nonradiative voltage loss $\left(\Delta V_{\mathrm{oc}, \mathrm{nrad}}\right)$ for the above device series using eq 8 and $V_{\text {oc,rad }}$ (from Table 2) and hence $\mathrm{QE}_{\mathrm{LED}}$ for the different devices in the series. From Table 2, we note that $V_{\text {oc,rad }}$ increases by more than $100 \mathrm{mV}$ across the series, whereas $V_{\text {oc }}$ increases by only $60 \mathrm{mV}$, from 423 to $485 \mathrm{mV}$. This leads to a $\Delta V_{\text {oc,nrad }}$ that is higher by more than $60 \mathrm{mV}$ in device $\mathrm{D}$ compared to device $\mathrm{A}$, and consequently a lower $\mathrm{QE}_{\mathrm{LED}}$ (Table 2). In other words, a larger fraction of charge carriers recombine radiatively rather than nonradiatively in device $A$ as compared to device D. From these results we can say that the CZTSe absorber with the lowest Sn content (device A) has a $V_{\text {oc }}$ that is the closest to its radiative limit among this series. Moreover, device A shows the highest $\mathrm{QE}_{\mathrm{LED}}$ of the series with a value around $5 \times 10^{-5} \%$, which is among the highest reported values for CZTSe devices ${ }^{36,37}$ but remains several orders of magnitude lower than typical values for CIGS or silicon solar cells which are $0.03 \%$ and $0.13 \%$, respectively. ${ }^{38}$

The significantly lower $\mathrm{QE}_{\mathrm{LED}}$ of our investigated CZTSe devices compared to CIGS or silicon suggests enhanced nonradiative recombination. Hence, to further investigate what controls $\mathrm{QE}_{\mathrm{LED}}$ for CZTS devices, we proceeded to use Cf-T and JV-T to understand the trend observed with changing $\mathrm{Sn}$ content.

Factors Affecting $Q E_{L E D}$. Measuring the temperature dependence of the capacitance of the devices at different frequencies is a commonly used method in the literature to characterize defects in chalcogenide materials or the properties of the interfaces with other layers. ${ }^{39-41}$ In this work we have measured $\mathrm{Cf}-\mathrm{T}$ for the four devices in the series, at temperatures ranging from 123 to $323 \mathrm{~K}$. Figure 2a shows the $\mathrm{Cf}-\mathrm{T}$ curves for device $\mathrm{A}$, while the curves for the other devices are presented in the Supporting Information (Figure S5). The inflection point frequency $\left(f_{t}\right)$ was subsequently extracted from the C-f curves for different temperatures for all the devices of the series. In previous work $f_{\mathrm{t}}$ is assumed to relate to the emission coefficient of the defect state and therefore to its energy. ${ }^{41,42}$ Thus, we were able to extract the activation energy of the dominant trap state in a ShockleyRead-Hall representation $\left(E_{\mathrm{a}, \mathrm{CfT}}\right)$ from the slope of an Arrhenius plot of $f_{\mathrm{t}}$ (Figure $2 \mathrm{~b}$ ). As kesterite absorbers are p-type, $E_{\mathrm{a}, \mathrm{CfT}}$ is considered to be the energy difference between the trap energy $\left(E_{t}\right)$ and the valence band maximum energy $\left(E_{\mathrm{v}}\right)$, which can therefore be written as $E_{\mathrm{a}, \mathrm{CfT}}=E_{\mathrm{t}}-E_{\mathrm{v}}$. Figure 3a shows $E_{\mathrm{a}, \mathrm{CfT}}$ calculated using this procedure for all the devices, together with the band gap extracted from the EL spectra. Clearly, the relative energy of the dominant trap state around $0.1 \mathrm{eV}$ does not change significantly with $\mathrm{Sn}$ content. Similar behavior is observed by Larramona et al. ${ }^{28}$ where the activation energy of a shallow defect with energy between 120 and $170 \mathrm{meV}$ is not correlated with $\mathrm{Sn}$ content. Further, apparent carrier concentration profiles were extracted from capacitance-voltage $(\mathrm{CV})$ measurements at room temperature for devices A, C and D (Figure S7, device B data are unavailable) with a voltage sweep from -1 to $+0.5 \mathrm{~V}$. The profiles are comparable for all the devices, showing a doping concentration on the order of $10^{16} \mathrm{~cm}^{-3}$ at $0 \mathrm{~V}$. The measured 

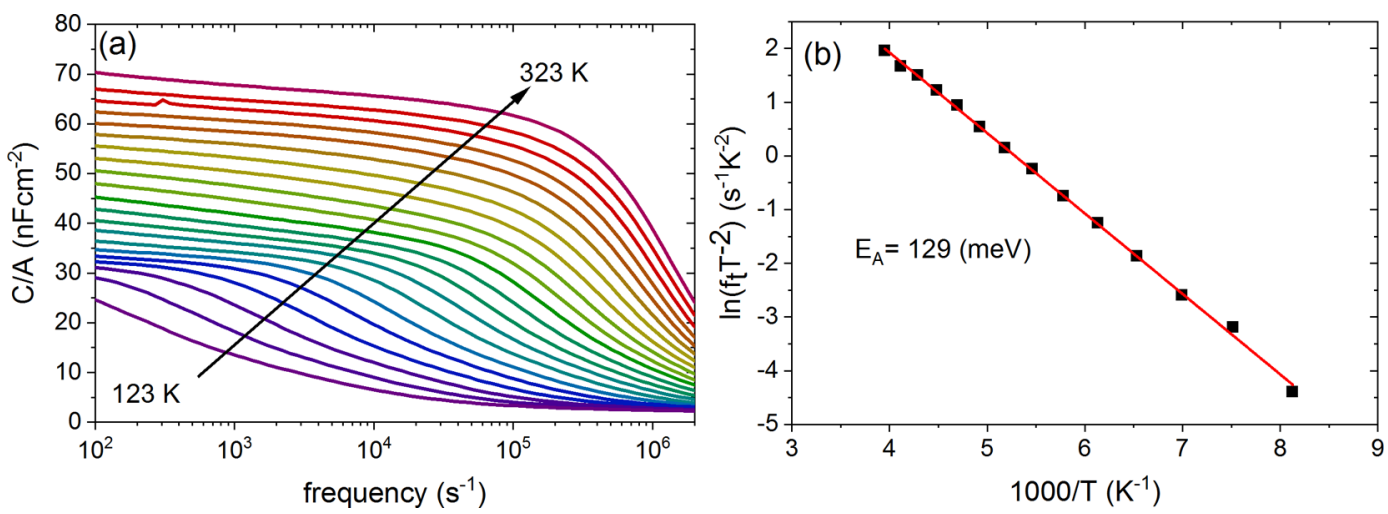

Figure 2. (a) Representative temperature-dependent capacitance-density frequency measurements in the temperature range from 123 to $323 \mathrm{~K}$ with $10 \mathrm{~K}$ steps and frequencies from $100 \mathrm{~Hz}$ to $1 \mathrm{MHz}$ of device A (Sn content of 27.6 at. \%). (b) Arrhenius plot of the inflection frequencies for device A.
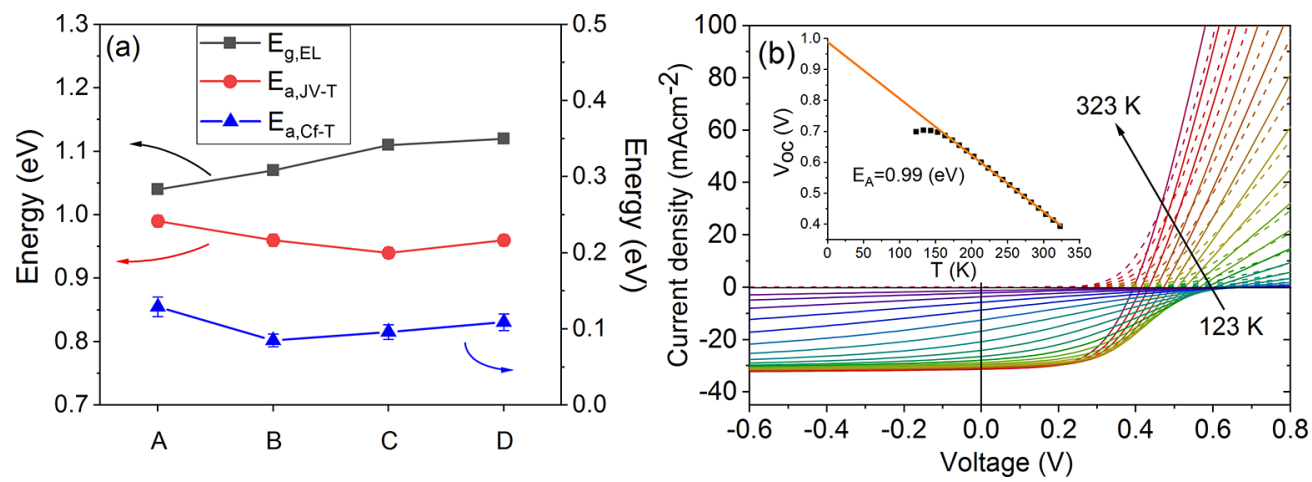

Figure 3. (a) Comparison among band gap energy $\left(E_{\mathrm{g}, \mathrm{EL}}\right)$ as obtained from EL measurements, activation energy of the main recombination mechanism $\left(E_{\mathrm{a}, \mathrm{JVT}}\right)$ obtained by JVT measurements, and activation energy of the main defect $\left(E_{\mathrm{a}, \mathrm{CfT}}\right)$ obtained by admittance spectroscopy for increasing Sn content. (The measurement error is too small to be properly visualized by the error bars.) (b) Temperature-dependent JV measurement (dark curve: dotted line, light curve: solid line) of device A (Sn content of 27.6 at. \%). The inset shows a linear fit of $V_{\text {oc }}$ extrapolated to $T=0 \mathrm{~K}$; a fitting error of $1 \%$ is taken into account.

trap state is therefore believed to be related to the defect chemistry in the near-surface region or a transport barrier as reported by Werner et al. ${ }^{43}$ Deep defects were not detected with the configuration for capacitance measurements presented here.

Note that defect densities and energies are usually expected to strongly affect $\mathrm{QE}_{\mathrm{LED}}$ of the device; however, in the studied series $\mathrm{QE}_{\mathrm{LED}}$ changed significantly with $\mathrm{Sn}$ content, while the trap states properties remained unchanged. This observation suggests that the measured change in $\mathrm{QE}_{\mathrm{LED}}$ with $\mathrm{Sn}$ content results not from trap states in the absorber inferred from $\mathrm{Cf}-\mathrm{T}$ in these devices, but from a different cause.

Finally, in order to investigate this further, we carried out temperature-dependent JV measurements for device A under AM1.5G illumination (Figure $3 \mathrm{~b}$ ), with similar plots for the rest of the series shown in Supporting Information Figure S6. The crossover of illuminated and dark JV-T curves becomes more pronounced at lower temperatures, whereas the increasing rollover leads to a complete blocking of the current at the lowest temperature of $123 \mathrm{~K}$. The inset shows the temperature dependence of the $V_{\mathrm{oc}}$, where the linear extrapolation to $0 \mathrm{~K}$ provides the activation energy of the dominant recombination mechanism $E_{\mathrm{a}, \mathrm{JVT}}{ }^{44}$ For device $\mathrm{A}$ the obtained $E_{\mathrm{a}, \mathrm{JVT}}=0.99 \mathrm{eV}$ is close to the derived bandgap $E_{\mathrm{g}, \mathrm{EL}}$ $=1.04 \mathrm{eV}$; thus the dominant recombination is within the bulk of the absorber rather than at the interface. While the band gap increases with $\mathrm{Sn}$ content, $E_{\mathrm{a}, \mathrm{JVT}}$ decreases slightly. Possible reasons for this are either worsened interface quality, due to the presence of domains of antibonding boundaries ${ }^{45,46}$ shunting paths on the nanoscale, ${ }^{47}$ or worsened band alignment, specifically with the CdS buffer layer as $\mathrm{Sn}$ is expected to mainly affect the conduction band minimum of the absorber. The latter is supported by first-principles studies showing that the conduction band minimum is dominated by antibonding Sn s and Se p hybrid orbitals while the valence band minimum does not involve Sn orbitals; thus a variation in Sn content will predominantly influence the conduction band. ${ }^{48,49}$ Device A shows the smallest difference between $E_{\mathrm{g}, \mathrm{EL}}$ and $E_{\mathrm{a}, \mathrm{JVT}}$ values, which amounts to about $0.05 \mathrm{eV}$. This means that the main recombination mechanism has an energy closer to the band gap of the absorber, thus a better band alignment with the buffer layer, than the devices of higher $\mathrm{Sn}$ content. $^{50-52}$ An increasing mismatch of the absorber layer and buffer layer conduction bands could explain the decreased $\mathrm{QE}_{\mathrm{LED}}$ with $\mathrm{Sn}$ content through increasing nonradiative losses via surface recombination at the interface with the buffer layer.

The present study highlights that quantifying the $V_{\text {oc }}$ loss as the difference between the measured device $V_{o c}$ and the band gap of the absorber is not the best metric for determining the voltage losses in compositionally complex absorbers like kesterites. By using two different methods to determine the bandgap, using either the absorption or emission spectra of the devices, we showed that the different methods lead to very different values in the $V_{\text {oc }}$ deficit relative to the bandgap, and 
quantitatively different $V_{\mathrm{oc}}$ loss trend in a series of solar cells with varying $\mathrm{Sn}$ content. Further, we show that by instead using the radiative limit to the open circuit voltage $\left(V_{\text {oc,rad }}\right)$ calculated from electroluminescence (EL) and external quantum efficiency (EQE) measurements, we can more reliably compare the voltage losses for the different devices in the series and subsequently quantify the electroluminescence quantum efficiency $\left(\mathrm{QE}_{\mathrm{LED}}\right)$ of the different devices. Interestingly, for this series of cells, the $V_{\text {oc }}$ increases with increasing $\mathrm{Sn}$ content from $423 \mathrm{mV}$ to $465 \mathrm{mV}$, while the $\mathrm{QE}_{\mathrm{LED}}$ decreases from $5 \times 10^{-5} \%$ to $5 \times 10^{-6} \%$. This result shows that while increasing $\mathrm{Sn}$ content increases device $V_{\text {oc }}$ it actually degrades the radiative efficiency of the device, increasing the loss in $V_{\text {oc }}$ due to nonradiative recombination. Regarding the origin of this effect, the decrease in $\mathrm{QE}_{\mathrm{LED}}$ appears not to be related to the defect properties of the absorber as no significant change in defect energy activation was observed. In fact, the solar cell with the best $\mathrm{QE}_{\mathrm{LED}}$ shows the best interface quality of the series as inferred from the JV-T measurement. Further investigations are needed to fully understand what controls $\mathrm{QE}_{\mathrm{LED}}$ in kesterite solar cells and how it is linked to the overall power conversion efficiency of the devices. We anticipate that this type of analysis will prove useful in analyzing the impact of material composition, processing, and device architecture on the voltage losses in other varieties of thin-film solar cells.

\section{ASSOCIATED CONTENT}

\section{S Supporting Information}

The Supporting Information is available free of charge on the ACS Publications website at DOI: 10.1021/acs.jpclett.9b00506.

Experimental section, XRF and XRD of the different absorbers, device perfomance data, EQE spectra, electroluminecence spectra fitting procedure, complementary voltage losses analysis, temperature dependent capacitance-frequency $(\mathrm{Cf}-\mathrm{T})$ plots for the remaining devices, temperature-dependent current density-voltage plots for the remaining devices, capacitance voltage measurements of different devices, and full PL spectra (PDF)

\section{AUTHOR INFORMATION}

\section{Corresponding Author}

*E-mail: jenny.nelson@imperial.ac.uk.

ORCID $\odot$

Mohammed Azzouzi: 0000-0001-5190-9984

\section{Author Contributions}

${ }^{\#}$ These authors contributed equally

\section{Notes}

The authors declare no competing financial interest.

\section{ACKNOWLEDGMENTS}

M.A. thanks the UK Engineering and Physical Sciences Research Council (ESPRC) for a postgraduate studentship. J.N. acknowledges funding from the EPSRC (grant numbers $\mathrm{EP} / \mathrm{P} 005543 / 1, \mathrm{EP} / \mathrm{M} 025020 / 1)$, the EPSRC Supersolar Hub (EP/P02484X/1), and the European Research Council under the European Union's Horizon 2020 research and innovation program (grant agreement No 742708). A.C.-V. acknowledges funding from Horizon 2020 program under the project
STARCELL (H2020-NMBP-03-2016-720907). The authors thank Flurin Eisner and Xingyuan Shi for their help with the measurement and their feedbacks.

\section{REFERENCES}

(1) Siebentritt, S.; Schorr, S. Kesterites-a Challenging Material for Solar Cells. Prog. Photovoltaics 2012, 20 (5), 512-519.

(2) Wang, W.; Winkler, M. T.; Gunawan, O.; Gokmen, T.; Todorov, T. K.; Zhu, Y.; Mitzi, D. B. Device Characteristics of CZTSSe ThinFilm Solar Cells with 12.6\% Efficiency. Adv. Energy Mater. 2014, 4 (7), 1301465 .

(3) Green, M. A.; Hishikawa, Y.; Dunlop, E. D.; Levi, D. H.; HohlEbinger, J.; Ho-Baillie, A. W. Y. Solar Cell Efficiency Tables (Version 52). Prog. Photovoltaics 2018, 26 (7), 427-436.

(4) Shockley, W.; Queisser, H. Detailed Balance Limit of Efficiency of P-n Junction Solar Cells. J. Appl. Phys. 1961, 32, 510-519.

(5) Rey, G.; Redinger, A.; Sendler, J.; Weiss, T. P.; Thevenin, M.; Guennou, M.; El Adib, B.; Siebentritt, S. The band gap of Cu2ZnSnSe4: Effect of order-disorder. Appl. Phys. Lett. 2014, 105, 112106.

(6) Redinger, A.; Unold, T. (SI) High Surface Recombination Velocity Limits Quasi-Fermi Level Splitting in Kesterite Absorbers. Sci. Rep. 2018, 1874.

(7) Haass, S. G.; Andres, C.; Figi, R.; Schreiner, C.; Bürki, M.; Romanyuk, Y. E.; Tiwari, A. N. (SI) Complex Interplay between Absorber Composition and Alkali Doping in High-Efficiency Kesterite Solar Cells. Adv. Energy Mater. 2018, 8, 1701760.

(8) Redinger, A.; Siebentritt, S. Loss Mechanisms in Kesterite Solar Cells. In Copper Zinc Tin Sulfide-Based Thin-Film Solar Cells; John Wiley \& Sons Ltd., 2014; pp 363-386.

(9) Repins, I. L.; Li, J. V.; Kanevce, A.; Perkins, C. L.; Steirer, K. X.; Pankow, J.; Teeter, G.; Kuciauskas, D.; Beall, C.; Dehart, C.; et al. Effects of Deposition Termination on Cu2ZnSnSe4device Characteristics. Thin Solid Films 2015, 582, 184-187.

(10) Mitzi, D. B.; Gunawan, O.; Todorov, T. K.; Wang, K.; Guha, S. The Path towards a High-Performance Solution-Processed Kesterite Solar Cell. Sol. Energy Mater. Sol. Cells 2011, 95 (6), 1421-1436.

(11) Sperling, R. A.; Parak, W. J.; Ackerson, C. J.; Jadzinsky, P. D.; Kornberg, R. D.; Akerman, M. E.; Chan, W. C. W.; Laakkonen, P.; Bhatia, S. N.; Ruoslahti, E.; et al. Surface Modification, Functionalization and Bioconjugation of Colloidal Inorganic Nanoparticles. Philos. Trans. R. Soc., A 2010, 368 (1915), 1333-1383.

(12) Romero, M. J.; Du, H.; Teeter, G.; Yan, Y.; Al-Jassim, M. M. Comparative Study of the Luminescence and Intrinsic Point Defects in the Kesterite $\mathrm{Cu} 2 \mathrm{ZnSnS} 4$ and Chalcopyrite $\mathrm{Cu}(\mathrm{In}, \mathrm{Ga}) \mathrm{Se} 2$ Thin Films Used in Photovoltaic Applications. Phys. Rev. B: Condens. Matter Mater. Phys. 2011, 84 (16), 1-5.

(13) Gokmen, T.; Gunawan, O.; Todorov, T. K.; Mitzi, D. B. Band Tailing and Efficiency Limitation in Kesterite Solar Cells. Appl. Phys. Lett. 2013, 103 (10), 103506

(14) Shin, D.; Saparov, B.; Mitzi, D. B. Defect Engineering in Multinary Earth-Abundant Chalcogenide Photovoltaic Materials. $A d v$. Energy Mater. 2017, 7 (11), 1602366.

(15) Chen, S.; Walsh, A.; Gong, X. G.; Wei, S. H. Classification of Lattice Defects in the Kesterite Cu2ZnSnS 4 and Cu2ZnSnSe4 EarthAbundant Solar Cell Absorbers. Adv. Mater. 2013, 25 (11), 15221539.

(16) Kumar, M.; Dubey, A.; Adhikari, N.; Venkatesan, S.; Qiao, Q. Strategic Review of Secondary Phases, Defects and Defect-Complexes in Kesterite CZTS-Se Solar Cells. Energy Environ. Sci. 2015, 8 (11), 3134-3159.

(17) Bourdais, S.; Choné, C.; Delatouche, B.; Jacob, A.; Larramona, G.; Moisan, C.; Lafond, A.; Donatini, F.; Rey, G.; Siebentritt, S.; et al. Is the $\mathrm{Cu} / \mathrm{Zn}$ Disorder the Main Culprit for the Voltage Deficit in Kesterite Solar Cells? Adv. Energy Mater. 2016, 6 (12), 1502276.

(18) Rey, G.; Redinger, A.; Sendler, J.; Weiss, T. P.; Thevenin, M.; Guennou, M.; El Adib, B.; Siebentritt, S. The Band Gap of 
Cu2ZnSnSe4: Effect of Order-Disorder. Appl. Phys. Lett. 2014, 105, 112106.

(19) Márquez, J.; Neuschitzer, M.; Dimitrievska, M.; Gunder, R.; Haass, S.; Werner, M.; Romanyuk, Y. E.; Schorr, S.; Pearsall, N. M.; Forbes, I. Systematic Compositional Changes and Their Influence on Lattice and Optoelectronic Properties of $\mathrm{Cu} 2 \mathrm{ZnSnSe} 4$ Kesterite Solar Cells. Sol. Energy Mater. Sol. Cells 2016, 144, 579-585.

(20) Krammer, C.; Huber, C.; Zimmermann, C.; Lang, M.; Schnabel, T.; Abzieher, T.; Ahlswede, E.; Kalt, H.; Hetterich, M. Reversible Order-Disorder Related Band Gap Changes in Cu2ZnSn(S,Se) 4 via Post-Annealing of Solar Cells Measured by Electroreflectance. Appl. Phys. Lett. 2014, 105 (26), 262104.

(21) Carron, R.; Andres, C.; Avancini, E.; Feurer, T.; Nishiwaki, S.; Pisoni, S.; Fu, F.; Lingg, M.; Romanyuk, Y. E.; Buecheler, S.; et al. Bandgap of Thin Film Solar Cell Absorbers: A Comparison of Various Determination Methods. Thin Solid Films 2019, 669 (9), 482-486.

(22) Rau, U.; Paetzold, U. W.; Kirchartz, T. Thermodynamics of Light Management in Photovoltaic Devices. Phys. Rev. B: Condens. Matter Mater. Phys. 2014, 90 (3), 1-16.

(23) Yao, J.; Kirchartz, T.; Vezie, M. S.; Faist, M. A.; Gong, W.; He, Z.; Wu, H.; Troughton, J.; Watson, T.; Bryant, D.; et al. Quantifying Losses in Open-Circuit Voltage in Solution-Processable Solar Cells. Phys. Rev. Appl. 2015, 4 (1), 1-10.

(24) Tuladhar, S. M.; Azzouzi, M.; Delval, F.; Yao, J.; Guilbert, A. A. Y.; Kirchartz, T.; Montcada, N. F.; Dominguez, R.; Langa, F.; Palomares, E.; et al. Low Open-Circuit Voltage Loss in SolutionProcessed Small-Molecule Organic Solar Cells. ACS Energy Lett. 2016, 1 (1), 302-308.

(25) Qian, D.; Zheng, Z.; Yao, H.; Tress, W.; Hopper, T. R.; Chen, S.; Li, S.; Liu, J.; Chen, S.; Zhang, J.; et al. Design Rules for Minimizing Voltage Losses in High-Efficiency Organic Solar Cells. Nat. Mater. 2018, 17, 703.

(26) Nelson, J. The Physics of Solar Cells; published by Imperial College Press and distributed by World Scientific Publishing Co., 2003 .

(27) Ki, W.; Hillhouse, H. W. Earth-Abundant Element Photovoltaics Directly from Soluble Precursors with High Yield Using a Non-Toxic Solvent. Adv. Energy Mater. 2011, 1 (5), 732-735.

(28) Larramona, G.; Levcenko, S.; Bourdais, S.; Jacob, A.; Choné, C.; Delatouche, B.; Moisan, C.; Just, J.; Unold, T.; Dennler, G. FineTuning the Sn Content in CZTSSe Thin Films to Achieve 10.8\% Solar Cell Efficiency from Spray-Deposited Water-Ethanol-Based Colloidal Inks. Adv. Energy Mater. 2015, 5 (24), 1501404.

(29) Ashley, T.; Elliott, C. T.; Gordon, N. R.; Hall, R. S.; Maxey, C. D.; Matthews, B. E. Room-Temperature Electroluminescence at Wavelengths of 5-7 Mm from $\mathrm{HgCdTe}$ Heterostructure Diodes. Appl. Phys. Lett. 1994, 65 (18), 2314-2316.

(30) Yakushev, M. V.; Sulimov, M. A.; Márquez-Prieto, J.; Forbes, I.; Krustok, J.; Edwards, P. R.; Zhivulko, V. D.; Borodavchenko, O. M.; Mudryi, A. V.; Martin, R. W. Influence of the Copper Content on the Optical Properties of CZTSe Thin Films. Sol. Energy Mater. Sol. Cells 2017, 168 (January), 69-77.

(31) Huang, D.; Persson, C. Band Gap Change Induced by Defect Complexes in Cu2ZnSnS4. Thin Solid Films 2013, 535, 265-269.

(32) Haass, S. G.; Andres, C.; Figi, R.; Schreiner, C.; Bürki, M.; Romanyuk, Y. E.; Tiwari, A. N. Complex Interplay between Absorber Composition and Alkali Doping in High-Efficiency Kesterite Solar Cells. Adv. Energy Mater. 2018, 8 (4), 1701760.

(33) Müller, T. C. M.; Pieters, B. E.; Kirchartz, T.; Carius, R.; Rau, U. Effect of Localized States on the Reciprocity between Quantum Efficiency and Electroluminescence in $\mathrm{Cu}(\mathrm{In}, \mathrm{Ga}) \mathrm{Se} 2$ and $\mathrm{Si}$ ThinFilm Solar Cells. Sol. Energy Mater. Sol. Cells 2014, 129, 95-103.

(34) Hages, C. J.; Carter, N. J.; Agrawal, R. Generalized Quantum Efficiency Analysis for Non-Ideal Solar Cells: Case of Cu2ZnSnSe4. J. Appl. Phys. 2016, 119 (1), 014505.

(35) Katahara, J. K.; Hillhouse, H. W. Quasi-Fermi Level Splitting and Sub-Bandgap Absorptivity from Semiconductor Photoluminescence. J. Appl. Phys. 2014, 116 (17), 173504.
(36) Yamaguchi, M.; Yamada, H.; Katsumata, Y.; Lee, K. H.; Araki, K.; Kojima, N. Efficiency Potential and Recent Activities of HighEfficiency Solar Cells. J. Mater. Res. 2017, 32 (18), 3445-3457.

(37) Green, M. A. Radiative Efficiency of State-of-the-art Photovoltaic Cells. Prog. Photovoltaics 2012, 20, 472-476.

(38) Kirchartz, T.; Rau, U.; Kurth, M.; Mattheis, J.; Werner, J. H. Comparative Study of Electroluminescence from $\mathrm{Cu}(\mathrm{In}, \mathrm{Ga}) \mathrm{Se} 2$ and $\mathrm{Si}$ Solar Cells. Thin Solid Films 2007, 515 (15 SPEC. ISS), 6238-6242. (39) Walter, T.; Herberholz, R.; Müller, C.; Schock, H. W. Determination of Defect Distributions from Admittance Measurements and Application to $\mathrm{Cu}(\mathrm{In}, \mathrm{Ga}) \mathrm{Se} 2$ Based Heterojunctions. J. Appl. Phys. 1998, 80 (8), 4411.

(40) Proskuryakov, Y. Y.; Durose, K.; Taele, B. M.; Oelting, S. Impedance Spectroscopy of Unetched $\mathrm{CdTe} / \mathrm{CdS}$ Solar Cellsequivalent Circuit Analysis. J. Appl. Phys. 2007, 102 (2), 024504.

(41) Gunawan, O.; Gokmen, T.; Warren, C. W.; Cohen, J. D.; Todorov, T. K.; Barkhouse, D. A. R.; Bag, S.; Tang, J.; Shin, B.; Mitzi, D. B. Electronic Properties of the $\mathrm{Cu} 2 \mathrm{ZnSn}(\mathrm{Se}, \mathrm{S}) 4$ Absorber Layer in Solar Cells as Revealed by Admittance Spectroscopy and Related Methods. Appl. Phys. Lett. 2012, 100 (25), 253905.

(42) Weiss, T. P. Electrical Characterization of Kesterite Thin Film Absorbers and Solar Cells; University of Luxembourg, 2015.

(43) Werner, F.; Siebentritt, S. Buffer Layers, Defects, and the Capacitance Step in the Admittance Spectrum of a Thin-Film Solar Cell. Phys. Rev. Appl. 2018, 9 (5), 54047.

(44) Nadenau, V.; Rau, U.; Jasenek, A.; Schock, H. W.; Nadenau, V.; Rau, U.; Jasenek, A.; Schock, H. W. Electronic Properties of CuGaSe 2 -Based Heterojunction Solar Cells. Part I. Transport Analysis. J. Appl. Phys. 2000, 87, 584.

(45) Park, J.-S.; Kim, S.; Walsh, A. Opposing Effects of Stacking Faults and Antisite Domain Boundaries on the Conduction Band Edge in Kesterite Quaternary Semiconductors. Phys. Rev. Materials 2018, 2, 014602.

(46) Kattan, N. A.; Griffiths, I. J.; Cherns, D.; Fermín, D. J. Observation of Antisite Domain Boundaries in Cu2ZnSnS4 by Atomic-Resolution Transmission Electron Microscopy. Nanoscale 2016, 8 (30), 14369-14373.

(47) Tiwari, D.; Cattelan, M.; Harniman, R.; Saruna, A.; Abbas, A.; Bowers, J. W.; Fox, N. A.; Fermin, D. J. Mapping Shunting Paths at the Surface of $\mathrm{Cu} 2 \mathrm{ZnSn}(\mathrm{S}, \mathrm{Se}) 4$ Films via Energy-Filtered Photoemission Microscopy. iScience 2018, 9, 36-46.

(48) Shu, Q.; Yang, J.-H.; Chen, S.; Huang, B.; Xiang, H.; Gong, X.G.; Wei, S.-H. Cu2Zn $(\mathrm{Sn}, \mathrm{Ge}) \mathrm{Se} 4$ and $\mathrm{Cu} 2 \mathrm{Zn}(\mathrm{Sn}, \mathrm{Si}) \mathrm{Se} 4$ Alloys as Photovoltaic Materials: Structural and Electronic Properties. Phys. Rev. B: Condens. Matter Mater. Phys. 2013, 87 (11), 115208.

(49) Chen, S.; Gong, X. G.; Walsh, A.; Wei, S.-H. Electronic Structure and Stability of Quaternary Chalcogenide Semiconductors Derived from Cation Cross-Substitution of II-VI and I-III-VI 2 Compounds. Phys. Rev. B: Condens. Matter Mater. Phys. 2009, 79, 165211.

(50) Haight, R.; Barkhouse, A.; Gunawan, O.; Shin, B.; Copel, M.; Hopstaken, M.; Mitzi, B.; Haight, R.; Barkhouse, A.; Gunawan, O. Band Alignment at the $\mathrm{Cu} 2 \mathrm{ZnSn}(\mathrm{SxSe} 1-\mathrm{x}) 4 / \mathrm{CdS}$ Interface. Appl. Phys. Lett. 2011, 98, 253502.

(51) Chen, S.; Walsh, A.; Yang, J.-H.; Gong, X. G.; Sun, L.; Yang, P.X.; Chu, J.-H.; Wei, S.-H. Compositional Dependence of Structural and Electronic Properties of $\mathrm{Cu} 2 \mathrm{ZnSn}(\mathrm{S}, \mathrm{Se}) 4$ Alloys for Thin Film Solar Cells. Phys. Rev. B: Condens. Matter Mater. Phys. 2011, 83 (12), 125201.

(52) Gao, S.; Jiang, Z.; Wu, L.; Ao, J.; Zeng, Y.; Sun, Y.; Zhang, Y. Interfaces of High-Efficiency Kesterite $\mathrm{Cu}_{2} \mathrm{ZnSnS}(\mathrm{e}){ }_{4}$ Thin Film Solar Cells. Chin. Phys. B 2018, 27 (1), 018803. 